\title{
Role of RHOB in the antiproliferative effect of glucocorticoid receptor on macrophage RAW264.7 cells
}

\author{
Xiaohui Wang*, Yuxia Chen*, Yan Wang, Xiaoyan Zhu, Yuanyuan Ma, Shimin Zhang ${ }^{\mathbf{1}}$ and Jian Lu \\ Department of Pathophysiology, Second Military Medical University, 800 Xiangyin Road, Shanghai 200433, People's Republic of China \\ ${ }^{1}$ American Registry of Pathology at Armed Forces Institute of Pathology, Washington, District of Columbia 20007, USA \\ (Correspondence should be addressed to J Lu; Email: lujian326@163.com) \\ *(X Wang and $\mathrm{Y}$ Chen are co-first authors)
}

\begin{abstract}
Although glucocorticoid (GC) has been reported to inhibit macrophage killing activity and cytokine production in response to proinflammatory stimuli, the effect of GC on macrophage proliferation is controversial. In our previous study, we found that inhibition of glucocorticoid receptor (GR) expression in murine macrophage cell line RAW264.7 cells (RAW-GR $(-$ ) cells) by RNAi significantly promoted cell proliferation. In the present study, we provide the evidence that the expression of Rhob, a member of Rho GTPases with anti-cancer character, remarkably decreased in RAW-GR(-) and RAW264.7 cells transiently transfected with GR-RNAi vector. Overexpression or constitutive activation of Rhob in RAW-GR(-) and RAW264.7 cells by transfection with wild-type Rhob expression vector (Rhob-wt) or constitutively activated Rhob plasmid (Rhob-V14) resulted in decreased proliferation of the two cell lines. Oppositely, the proliferation of RAW264.7 cells was
\end{abstract}

significantly increased when the expression of Rhob by RNA interference technique or the activity of Rhob by transfection with dominant negative Rhob mutant that is defective in nucleotide binding (Rhob-N19) was inhibited. In addition, enhanced activity of Akt, but not MAPK3/1 or MAPK14, was found in RAW-GR $(-)$ cells. Blocking the pathway of phosphatidylinositol 3-kinase (PI3K)/Akt with the specific inhibitor LY294002 decreased the proliferation and elevated RHOB protein level, indicating that PI3K/Akt signal plays its role of proliferation modulation upstream of RHOB protein. In conclusion, these results demonstrate that Rhob plays an important role in the antiproliferative effect of GR on RAW264.7 cells by GR $\rightarrow$ Akt $\rightarrow$ Rhob signaling and Rhob negatively regulates the proliferation of RAW264.7 cells.

Journal of Endocrinology (2009) 200, 35-43

\section{Introduction}

Glucocorticoid (GC) is a significant hormone in regulating diverse physiological processes such as immune and inflammation responses, cell proliferation, differentiation, and apoptosis (Dondi et al. 2001, Kudawara et al. 2001, Distelhorst 2002, Yamamoto et al. 2002). The common use of GC in treating inflammatory and autoimmune diseases is mostly due to its function of immunosuppression, for instance, inhibition of growth and induced apoptosis in lymphocytes, and repression of cytokine production in macrophages (Distelhorst 2002, Frankfurt \& Rosen 2004). Macrophages also play a key role in the immune and inflammatory process, mainly acting as antigen-processing/presenting cells and sources of inflammatory cytokines. Although GC has been reported to suppress macrophage killing activity and cytokine production in response to proinflammatory stimuli (Brummer et al. 2005), the effect of GC on macrophage proliferation is controversial (Lloberas et al. 1998, Sakai et al. 1999, Pagniello et al. 2002).

It is well known that the action of GC was mediated by glucocorticoid receptor (GR) that belongs to nuclear receptor superfamily and is normally localized in the cytoplasm in an inactive state. On hormone binding, GR translocates to the nucleus where it binds to glucocorticoid response elements (GREs) on target genes, recruits coactivators, or corepressors to regulate the expression of target genes (Heitzer et al. 2007). But a few target genes have been known to mediate the antiproliferative effect of GC until now. Rhob is a member of Rho GTPase family and has a tumor-suppressive role, including inhibiting cell proliferation and inducing apoptosis in several human cancer cells, and inhibiting tumor growth in a nude mouse xenograft model (Prendergast 2001, Mazieres et al. 2004). Rhob expression level has recently been shown to dramatically decrease in several cancer cells and patient biopsies such as lung cancer, brain cancer, neck and head tumors as tumors become more aggressive, and ectopic expression of Rhob antagonizes oncogene-mediated transformation (Liu et al. 2001, Adnane et al. 2002, Forget et al. 2002, Jiang et al. 2004a,b, Mazieres et al. 2004, Huang \& Prendergast 2006). Recently, we found that GC/GR increased Rhob expression in several tumor cells, such as human ovarian cancer cell lines HO-8910 and $3 \mathrm{AO}$, as well as human hepatocellular carcinoma cell 
SMMC-7721, and the proliferation inhibition of GC/GR on HO-8910 cells is partly due to the increased expression of $R$ hob (Chen et al. 2006). Although Rhob activation was required for mannose receptor-mediated phagocytosis of human alveolar macrophages (Zhang et al. 2005), the role of Rhob in the proliferation of macrophage was unclear until now.

In our previous study, we found that Dex inhibited the growth of RAW264.7 cells, a murine macrophage cell line. Treatment of RAW264.7 cells with $10^{-7} \mathrm{M}$ Dex for 4 days resulted in about $27 \%$ inhibition of cell proliferation. We also found unexpectedly that inhibition of GR expression in RAW264.7 by RNA interference significantly promoted cell proliferation. The cell number of RAW-GR $(-)$ cells (stably transfected with GR RNA interference plasmid) was almost twofold as that of the RAW-control cells (stably transfected with RNA interference control plasmid) after 4-day culture (Zhu et al. 2004). These results indicated that GC/GR negatively regulated the growth of RAW264.7 cell, and compared with GC, the expression of functional GR seemed to be more important in keeping cells a more differentiated and less proliferative phenotype. This prompted us to further clarify the molecular mechanism responsible for the antiproliferative effect of GR on RAW264.7 cells. In this study, we investigated the expression of Rhob in RAW-GR $(-)$ cells and its possible role in GC/GR-mediated proliferation inhibition of RAW264.7 cells.

\section{Materials and Methods}

\section{Materials}

Materials used in the present study included mouse monoclonal antibodies against $\beta$-actin and 3-(4,5-dimethylthiazol-2-yl)2,5-diphenyl-2H-tetrazolium bromide (MTT; Sigma-Aldrich Chemicals); rabbit polyclonal antibodies against GR and Rhob (Santa Cruz Biotechnology, Santa Cruz, CA, USA); goat antirabbit-AP and goat anti-mouse-AP conjugate (Bio-Rad Laboratories); RPMI 1640 medium (Life Technologies Inc.); RNA interfering expression vector pSilencer 2.1-U6 and negative control plasmid (Ambion, Austin, TX, USA); and BCA protein assay kit (Pierce, Pierce, IL, USA).

The wild-type Rhob expression vector (pcDNA3-Rhob; named $R$ hob-wt), constitutively active Rhob mutant containing a point mutation in the GTPase domain (G14V; named $R$ hob-V14), and dominant negative Rhob mutant that is defective in nucleotide binding (T19N; named Rhob-N19) were generously provided by Dr Prendergast (2001).

\section{Cell lines and cell culture}

Murine macrophage cell line RAW264.7 was obtained from American Type Culture Collection (Rockville, MD, USA). RAW-GR (-) and RAW-control cells were established by stable transfection of RAW264.7 cells with GR-siRNA expression vector and siRNA control vector respectively (Zhu et al. 2004). All the cells were cultured in RPMI 1640 medium containing $10 \%$ heat-inactivated fetal bovine serum at $37^{\circ} \mathrm{C}$ in a humidified incubator of $5 \% \mathrm{CO}_{2}$.

\section{Quantitative real-time PCR}

Total RNA was extracted with the TRIzol reagent and $2 \mu \mathrm{g}$ total RNA was used to synthesize first-strand cDNA by reverse transcription system (Promega), in accordance with the manufacturer's instructions.

Primers for amplification of Rhob gene were sense: $5^{\prime}-\mathrm{CTG}$ GCC CGC ATG AAG CA-3' and antisense: $5^{\prime}$-AGG CAG TCT GGT GGT GTC C-3' (186 bp), and those for $\beta$-actin were sense: $5^{\prime}$-CTG TAT GCC TCT GGT CGT AC-3' and antisense: $5^{\prime}$-TGA TGT CAC GCA CGA TTT CC-3' $(214 \mathrm{bp})$. SYBR Green from OPEN company was used as the fluorescence reagent. cDNA template (100 and $50 \mathrm{ng}$ for the amplification of $R h o b$ and $\beta$-actin respectively) was added to the mixture of $2 \mu \mathrm{l} 10 \times$ buffer, $1.6 \mu \mathrm{l}$ of $25 \mathrm{mM} \mathrm{MgCl}_{2}, 0.5 \mu \mathrm{l}$ of $20 \mathrm{mM}$ dNTP, $0 \cdot 3 \mu \mathrm{l}$ of $20 \mathrm{mM}$ primers, $1.5 \mathrm{U}$ Taq enzyme, and $1 \cdot 0 \mu \mathrm{l}$ of $20 \times$ SYBR Green to a final volume of $20 \mu \mathrm{l}$. Amplification of $R h o b$ and $\beta$-actin was performed in different tubes, with the same process: denaturation at $94{ }^{\circ} \mathrm{C}$ for $15 \mathrm{~s}$, annealing at $57^{\circ} \mathrm{C}$ for $20 \mathrm{~s}$, elongation at $72^{\circ} \mathrm{C}$ for $15 \mathrm{~s}$ for 36 cycles, and a final extension for $10 \mathrm{~min}$ at $72{ }^{\circ} \mathrm{C}$.

The mRNA ratio of Rhob and actin was calculated as follows: we first drew the standard curves of Rhob and $\beta$-actin by measuring the threshold cycles (CT) of standards of different concentrations, which had strong positive relationship with the start copy number (i.e. cDNA amounts) of a gene in linear amplification period. Then the cDNA amounts of $R h o b$ and $\beta$-actin of each testing sample were calculated according to their standard curves, and cDNA amount of $R$ hob was normalized by the corresponding $\beta$-actin.

\section{Western blot analysis}

Whole cell extract was prepared with lysis buffer (Stamatakis et al. 2002; $10 \mathrm{mM}$ Tris ( $\mathrm{pH} 7 \cdot 5$ ), $0 \cdot 1 \mathrm{mM}$ EDTA, $0.1 \mathrm{mM}$ EGTA, $0 \cdot 5 \%$ SDS, $0 \cdot 1 \mathrm{mM}$ mercaptoethanol, $2 \mu \mathrm{g} / \mathrm{ml}$ of each of the protease inhibitors leupeptin, aprotinin, and pepstatin). The lysate was briefly sonicated on ice, and spun at $14000 \mathrm{r} . \mathrm{p} . \mathrm{m} . / \mathrm{min}$ for $10 \mathrm{~min}$ to remove the insoluble material. Protein concentrations of the supernatant were measured with BCA protein assay kit. Twenty to fifty micrograms of extracts were resolved on 10\% SDS-PAGE gel. After electrophoresis, the proteins were electrotransferred to a nitrocellulose membrane, which were then blocked with $5 \%$ nonfat milk and probed overnight with antibodies against Rhob (1:200), GR (1:1000), total and phosphorylated MAPK3/1, MAPK14, phosphorylated Akt (1:500), or $\beta$-actin (1:10 000). Then the membranes were washed, exposed to alkaline phosphatase (AP)-conjugated secondary antibodies for $2 \mathrm{~h}$, and finally detected by NBT/BCIP staining. The quantitativeness of protein expression was made by Tanon Bio-image software (Shanghai, China). 
$R N A$ interference constructs for $\mathrm{Rhob}$ and transfection

Using online siRNA sequence selector tool (BD Clontech), siRNA templates were designed to match nonconserved 19 nucleotide sequences within the mouse Rhob mRNA. The sequences of the oligonucleotides used to create Rhob-RNAi

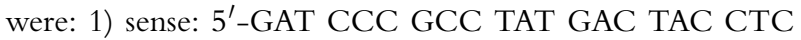
GAG TTT CAA GAG AAC TCG AGG TAG TCA TAG GCT TTT TTG GAA A-3' and antisense: $3^{\prime}$-GGC GGA TAC TGA TGG AGC TCA AAG TTC TCT TGA GCT CCA TCA GTA TCC GAA AAA A CC TTT TCG A-5 ${ }^{\prime}$, and 2) sense: 5'-GAT CCC GCG CTA CGG ATC CCA GAA TTT CAA GAG AAT TCT GGG ATC CGT AGC GCT TTT TTG GAA A-3' and antisense: $3^{\prime}$-GGC GCG ATG CCT AGG GTC TTA AAG TTC TCT TAA GAC CCT AGG CAT CGC GAA AAA ACC TTT TCG A-5'. Negative control plasmid was supplied from Ambion; it expressed a hairpin siRNA with limited homology to any known sequences in the human, mouse, and rat genomes. The oligonucleotides were synthesized by Sangon Technology Inc. (Shanghai, China) and were annealed/cloned into pSilencer 2.1-U6 vectors. The clones were confirmed by DNA sequencing. Expression of siRNA for Rhob is under control of the U6 promoter.

pSilencer 2.1-U6-Rhob or pSilencer 2.1-U6 control plasmid was introduced into RAW264.7 cells with LipofectAMINE Plus reagent (Invitrogen). Briefly, the cells were transfected with $3 \mu \mathrm{g}$ DNA in each well of a 6-well plate, according to the manufacturer's instructions.

\section{Transient transfection of the cells}

Cells were seeded onto 24 -well plates $\left(4 \times 10^{4}\right.$ cells/well) or 6 -well plates $\left(2 \times 10^{5}\right.$ cells/well) for $24 \mathrm{~h}$ and then transiently transfected with 1 (for 24-well plate) or $3 \mu \mathrm{g}$ (for 6-well plate) of the blank vector (pcDNA3), Rhob-wt, Rhob-V14, RhobRNAi, or Rhob-N19 plasmid using LipofectAMINE Plus reagent (Invitrogen), according to the manufacturer's instructions.

\section{Cell proliferation assay}

Twenty-four hours after transfection with various Rhob plasmid as indicated above, cells were rinsed out and seeded in duplicate at $3 \times 10^{4}$ cells per well onto 24 -well plate for MTT assay or $2 \times 10^{5}$ cells per well onto 6-well plate for cytometry. After incubation for 2 days, the cell number in each well was collected and counted with a hemacytometer or examined by MTT assay. Two hundred microliters of RPMI 1640 medium containing $5 \mathrm{mg} / \mathrm{ml}$ MTT (Sigma-Aldrich Chemicals) solution were added to each well. The cells were incubated for another $4 \mathrm{~h}$ at $37^{\circ} \mathrm{C}$, followed by solubilization with $200 \mu \mathrm{l}$ DMSO solutions. Then the O.D. value was measured using a Bio-Rad (model 550) microplate reader at $550 \mathrm{~nm}$ with the DMSO as blank.
The linear response range of RAW264.7 cells between the results of MTTassay and the number of cells was explored, and it was found that there was a linear correlation when the cells were seeded at the densities from $1 \times 10^{4}$ to $4 \times 10^{5}$ cells per well onto a 24-well plate and cultured in the medium for 4 days (data not shown). Therefore, in our experiments, $3 \times 10^{4}$ cells per well in a 24-well plate were selected, cultured in the medium for 4 days, and then cell proliferation was detected by MTT assay.

\section{Statistical analysis}

Data are expressed as $\mathrm{X} \pm$ S.D. Statistical significance was determined by Student's $t$-test with $P<0 \cdot 05$ considered as statistically significant.

\section{Results}

Inhibition of GR expression in RAW264.7 cells by stable or transient transfection with GR RNA interference vector decreased Rhob expression of the cells

Considering that $R$ hob showed a proliferation inhibitory effect on several tumor cells, and the proliferation of RAW-GR (-) cells (stably transfected with GR-RNAi vector) increased after inhibition of GR expression, we examined the expression of Rhob at mRNA and protein levels in RAW-GR $(-)$ cells by quantitative real-time PCR (Fig. 1A) and western blot analysis (Fig. 1B) respectively. As we expected, the mRNA and protein levels of Rhob in RAW-GR $(-)$ cells were reduced to about $22 \%$ and $28 \%$ of RAW-control cells respectively.

RAW-GR $(-)$ cells were originated from a single clone. Several single clones of RAW-GR $(-)$ cells were established and down-regulation of Rhob expression was also found in other clones (data not shown). To validate down-regulation of Rhob expression induced by a decreased level of GR, RAW264.7 cells were transient transfected with GR-RNAi vector and the protein level of Rhob was decreased in RAW264.7 cells (Fig. 1C), which indicated that downregulation of Rhob expression in RAW264.7 cells stably or transiently transfected with GR-RNAi vector was indeed a result from the knock down of GR.

Decreased expression of $\mathrm{Rhob}$ is involved in the increased proliferation of $R A W-G R(-)$ cell

As increased proliferation was in concurrence with significantly decreased expression of Rhob in RAW-GR(-) cells, we hypothesized that decreased expression of Rhob might be related to the growth acceleration of RAW-GR $(-)$ cells. To evaluate this possibility, we transfected RAW-GR $(-)$ cells with Rhob-wt (Rhob wild-type construct) Rhob-V14 (a constitutively active Rhob plasmid) or blank vector to detect the effect of Rhob on the cell proliferation by MTT assay and cytometry. As shown in Fig. 2A, the protein level of Rhob increased about twofold in RAW-GR $(-)$ cells transfected with Rhob-wt plasmid. The increase in expression and activation 
of Rhob in RAW-GR $(-)$ cells resulted in about 27 and $34 \%$ decrease in cell proliferation respectively (Fig. 2B). Similar results were obtained by cytometry (Fig. 2C). These data indicated that decreased expression of Rhob is involved in the increased proliferation of RAW-GR $(-)$ cell.

GC/GR induces the expression of $\mathrm{R}$ hob at $m R N A$ and protein levels in $R A W 264.7$ cells

Recently, we found that GC/GR increased Rhob expression in human ovarian cancer cell lines HO-8910 and 3AO, as well

A

Real-time PCR

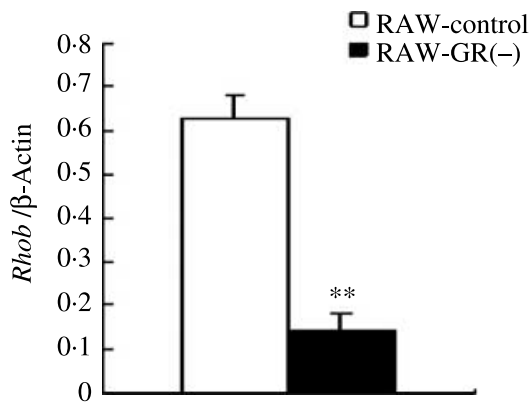

B

Western blot
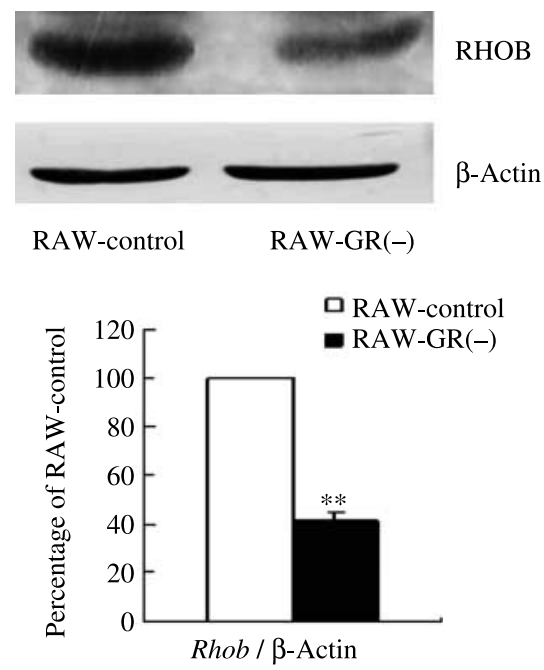

$\mathrm{C}$

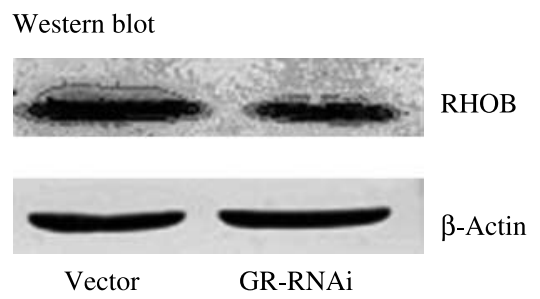

as human hepatocellular carcinoma cell SMMC-7721, and the proliferation inhibition of GC/GR on HO-8910 cells is partly due to the increased expression of Rhob (Chen et al. 2006). To determine the effect of GC on the expression of Rhob in RAW264.7 cells, we investigated the mRNA and protein levels of $R$ hob in the cells treated with $10^{-7} \mathrm{M}$ Dex for different times by quantitative real-time PCR and western blot respectively. Figure $3 \mathrm{~A}$ shows that Dex induced the mRNA expression of Rhob in a time-dependent manner, with the maximal effect at $2 \mathrm{~h}$ after treatment with $10^{-7} \mathrm{M}$ Dex. A significant increase in $\mathrm{RHOB}$ protein expression was also observed following incubation of cells with $10^{-7} \mathrm{M} \mathrm{Dex}$ for $4 \mathrm{~h}$. Furthermore, RU486, anti-agonist of GR, could partially attenuate the up-regulation of Rhob by Dex, indicating that activation of GR is required for Rhob induction (Fig. 3B).

$\mathrm{R}$ hob negatively regulates the proliferation of parental RAW264.7 cells

To examine whether Rhob has a direct inhibition effect on the proliferation of parental RAW264.7 cells, we used RNAi (RNA interference) to knock down endogenous Rhob expression in this cell line. The results of western blot showed that both the two Rhob-RNAi plasmids obviously reduced the expression of Rhob in RAW264.7 cells by about $73 \%$ [Rhob-RNAi(1)] and 88\% [Rhob-RNAi(2)], when compared with that of control siRNA plasmid (Fig. 4).

The proliferation of RAW264.7 cells was detected by MTT and cytometry after transient transfection with blank vector, Rhob-RNAi(2), Rhob-N19 (dominant negative mutant plasmid), Rhob-wt, or Rhob-V14. As shown in Fig. 5A, inhibition of the expression and the activity of $R h o b$ by transfection with Rhob-RNAi and Rhob-N19 resulted in about 44 and 36\% increase in the cell number respectively, compared with that of the blank vector control (pcDNA3 or RNAi-control) cells. Rhob overexpression or constitutive activation of Rhob by transfection of RAW264.7 cells with Rhob-wt or Rhob-V14 decreased the cell number to about 80 and $62 \%$ of the blank

Figure 1 Inhibition of GR expression in RAW264.7 cells by stable or transient transfection with GR RNA interference vector decreased Rhob expression of the cells. (A) Rhob mRNA level in RAW-GR(-) and RAW-control cells (stably transfected with GR-RNAi vector and RNAi control vector respectively) was detected by quantitative

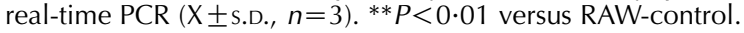
(B) The protein level of RHOB in the two cells was assayed by western blot analysis. Similar results were obtained from three independent experiments. Using Tanon software (Shanghai China), the blots of Rhob in these two cells were quantified and normalized against $\beta$-actin. The normalized numbers from three independent experiments were compared. The values are expressed as the percentage of the level of RAW-control, as shown in the bar graph of $\mathrm{B}(\mathrm{X} \pm$ s.D., $n=3)$. ${ }^{* *} P<0 \cdot 01$ versus RAW-control. (C) The protein level of RHOB in RAW264.7 cells transiently transfected with GR-RNAi vector or RNAi control vector was assayed by western blot analysis. Similar results were obtained from three independent experiments. 
A

1
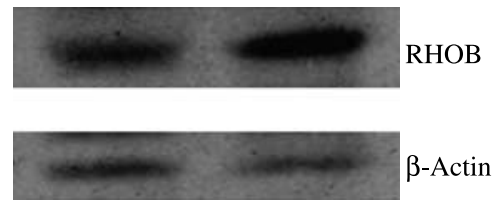

Vector

Rhob-wt
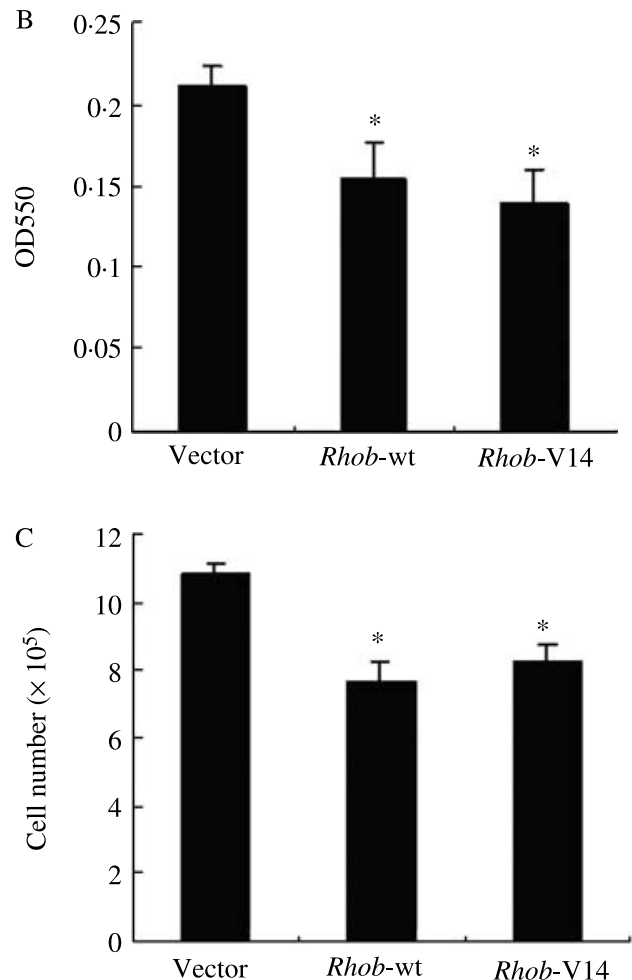

Figure 2 Transfection of RAW-GR(-) cells with Rhob wild-type plasmid or constitutively active Rhob plasmid inhibits cell proliferation. (A) The protein level of RHOB in RAW-GR $(-)$ cells was assayed by western blot analysis. Similar results were obtained from three independent experiments. RAW-GR $(-)$ cells were transfected with blank vector, Rhob-wt (wild-type Rhob cDNA), or Rhob-V14 (constitutively active Rhob mutant) for $24 \mathrm{~h}$, and then seeded and cultured in duplicate for 2 days onto 24 -well plate $\left(3 \times 10^{4}\right.$ cells/well) for (B) MTT assay or (C) 6 -well plate $\left(2 \times 10^{5}\right.$ cells/well) for cytometry (X土s.D.). ${ }^{*} P<0 \cdot 05$ versus cells transfected with blank vector. $n=3$.

vector control cells. Similar results were obtained by MTT assay (Fig. 5B). These results further demonstrated that Rhob signaling negatively regulated the proliferation of RAW264.7 cells.

Akt phosphorylation, but not MAPK3/1 and MAPK14, is involved in the accelerating the growth of $R A W-G R(-)$ cells

In recent years, evidence has emerged that GR plays important roles in regulating cell proliferation by modulating
A Real-time PCR

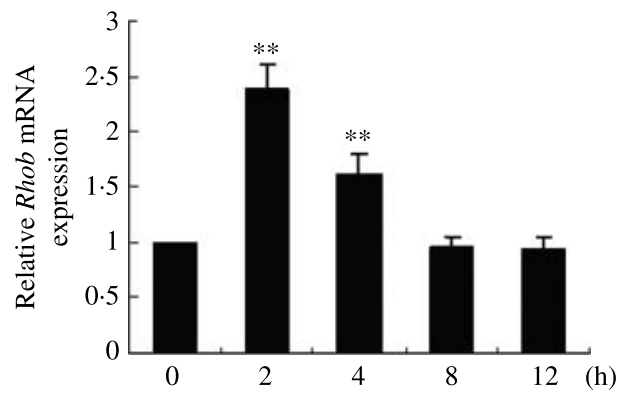

B Western blot
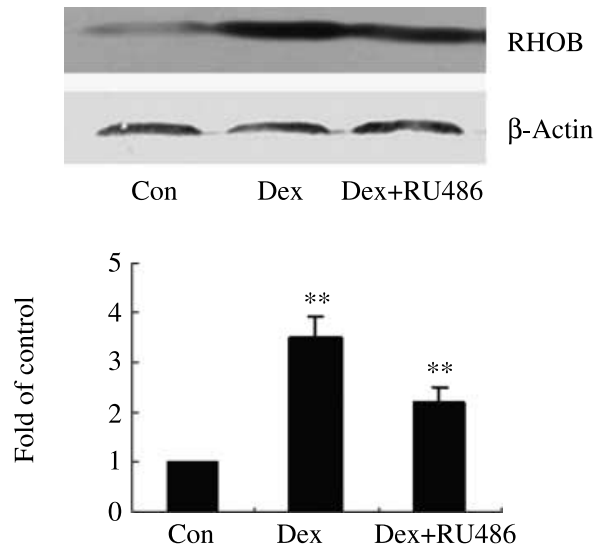

Figure 3 Dex increases $R h o b$ expression at mRNA and protein levels in RAW264.7 cells. (A) Rhob mRNA expression in RAW264.7 cells after treatment with $10^{-7} \mathrm{M}$ concentrations of Dex for different times was examined by real-time PCR. Dex induced the mRNA expression of Rhob in a time-dependent manner, with the maximal effect at $2 \mathrm{~h}$ after treatment with $10^{-7} \mathrm{M} \operatorname{Dex}(\mathrm{X} \pm$ s.D., $n=3$ ). ${ }^{* *} P<0 \cdot 01$ versus control. (B) Protein level of RHOB in RAW264.7 cells treated with $10^{-7} \mathrm{M}$ Dex with or without $10^{-6} \mathrm{M}$ RU486 was examined by western blot analysis. Similar results were obtained from three independent experiments. The blots of Rhob were quantified by Tanon software and normalized against $\beta$-actin. Then the normalized numbers from three independent experiments were compared. The values are expressed as fold of the level of control, as shown in the bar graph of (B) $(\mathrm{X} \pm$ s.D., $n=3) .{ }^{* *} P<0 \cdot 01$ versus control.

the activity of Akt (Leis et al. 2004, Chrysis et al. 2005, Nuutinen et al. 2006), MAPK3/1 and MAPK14 (Miller et al. 2005, Qin et al. 2005), so we examined the activated status of these kinases in RAW-GR $(-)$ and RAW264.7 cells by western blot. We failed to find a significant difference in phosphorylated MAPK3/1 or MAPK14 level between RAW-GR(-) and RAW-control cells (data not shown), but found about a 79\% increase in phosphorylated Akt in RAW-GR $(-)$ cells compared with that of RAW-control cells (Fig. 6A). To validate the effect of Akt on the increased proliferation of RAW-GR $(-)$ cells, we further examined the effect of LY294002, a specific inhibitor of phosphatidylinositol 3-kinase (PI3K)/Akt pathway, on cell growth by 
A
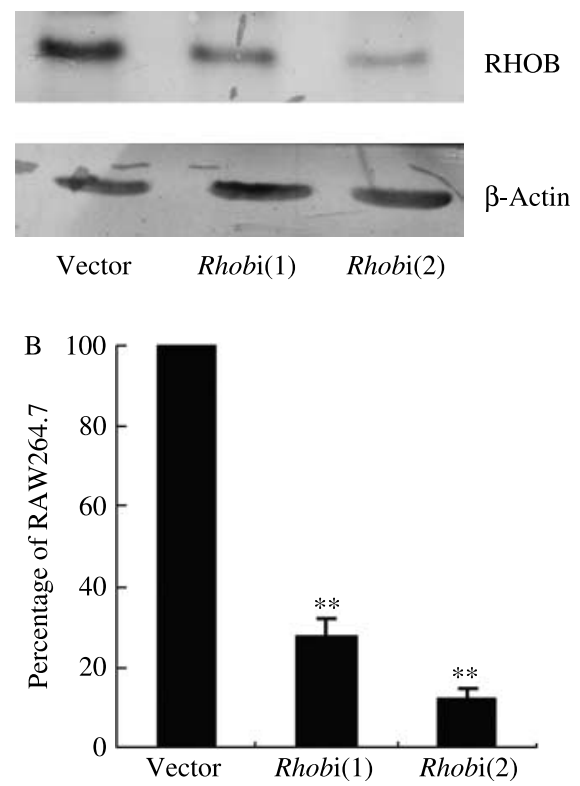

Figure 4 RNA interference target Rhob gene knocks down Rhob expression in RAW264.7 cells. Twenty-four hours after transfection, RAW264.7 cells with negative control vector, pSilencer 2.1-U6Rhobi(1), or pSilencer 2.1-U6-Rhobi(2), protein expression of $\mathrm{RHOB}$ in the cells was assayed by western blot analysis. Similar results were obtained from three independent experiments. The blots of RHOB in these two cells were quantified by Tanon software and normalized against $\beta$-actin. The normalized numbers from three independent experiments were compared. The values are expressed as the percentage of the level of RAW-control, as shown in the bar graph (X土s.D., $n=3$ ). ${ }^{* *} P<0 \cdot 01$ versus RAW264.7 cells transfected with negative control vector.

MTT assay. As shown in Fig. 6B, treatment of cells with $25 \mu \mathrm{M}$ LY294002 resulted in a more obvious growth repression in RAW-GR $(-)$ cells than that in RAW-control cells, though the effect could be seen in both the two cells. These results indicated that enhanced activity of Akt, but not MAPK3/1 and MAPK14, was also associated with the increased proliferation of RAW-GR $(-)$ cells.

\section{Blocking PI3K/Akt pathway in RAW-GR(-) and} RAW264.7 cells by LY294002 and inhibiting Akt activity in RAW264.7 cells by Dex-increased Rhob expression of the cells

Given that both PI3K/Akt and Rhob are involved in the growth of RAW-GR $(-)$ cells, the relevance between the two signaling pathways was then investigated. RAW-GR $(-)$ cells and its parental RAW264.7 cells were treated with $0-50 \mu \mathrm{M}$ LY294002 for $12 \mathrm{~h}$, and the expression of Rhob mRNA was then detected by real-time PCR. As shown in Fig. 7A, the expression of Rhob mRNA in RAW-GR (-) and RAW264.7 cells increased gradually with the dosage of LY294002, and the mRNA level of Rhob in RAW-GR (-) cells is about 2.3-
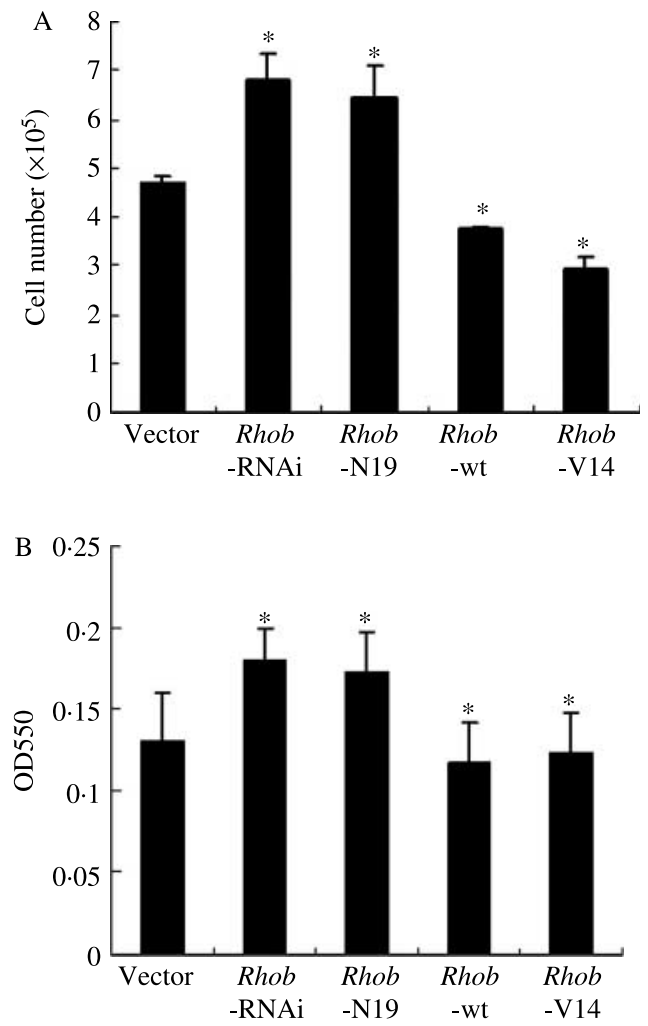

Figure 5 Increase in RHOB protein level or activity inhibits the proliferation of RAW264.7 cells, while decrease in RHOB protein level or activity promotes the cell proliferation. The level or activity of Rhob was increased by transfection of RAW264.7 cells with Rhob-wt (wild-type Rhob cDNA) or Rhob-V14 (constitutively active Rhob mutant), while the level or activity of Rhob was decreased by transfection with Rhob-RNAi (Rhob RNA interference plasmid) or Rhob-N19 (dominant negative mutant Rhob plasmid). Twenty-four hours after transfection, the cells were seeded and cultured in duplicate for 2 days in 6 -well plate $\left(2 \times 10^{5}\right.$ cells/well $)$ for (A) cytometry or (B) 24-well plate $\left(3 \times 10^{4}\right.$ cells/well) for MTT assay $(X \pm$ s.D. $)$.

${ }^{*} P<0 \cdot 05$ versus cells transfected with blank vector $(n=3)$.

and 3-2-fold of the control at 25 and $50 \mu \mathrm{M}$ LY294002 respectively. The expression of RHOB protein was also further examined. The cells were treated with or without $50 \mu \mathrm{M}$ LY294002, and the protein level was analyzed by western blot. Figure 7B shows that Rhob protein in RAWGR (-) and RAW264.7 cells increased remarkably when compared with that of control when $50 \mu \mathrm{M}$ LY294002 was added. These results demonstrated that PI3K/Akt signal was in the upstream of Rhob in these two cells and downregulation of Rhob in RAW-GR $(-)$ cells was resulted from the activation of PI3K/Akt pathway.

As shown in Fig. 3, we demonstrated that Dex increased the expression of Rhob in RAW264.7 cells at mRNA and protein levels. Does Dex also affect PI3K/Akt activity in RAW264.7 cells. Was the role of Dex on Rhob in the cells associated with Akt pathway? Therefore, we detected the effect of Dex on the protein level of p-Akt and Rhob in 
A

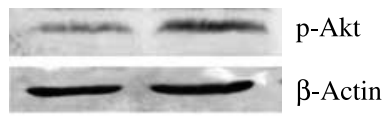

RAW-control RAW-GR(-)
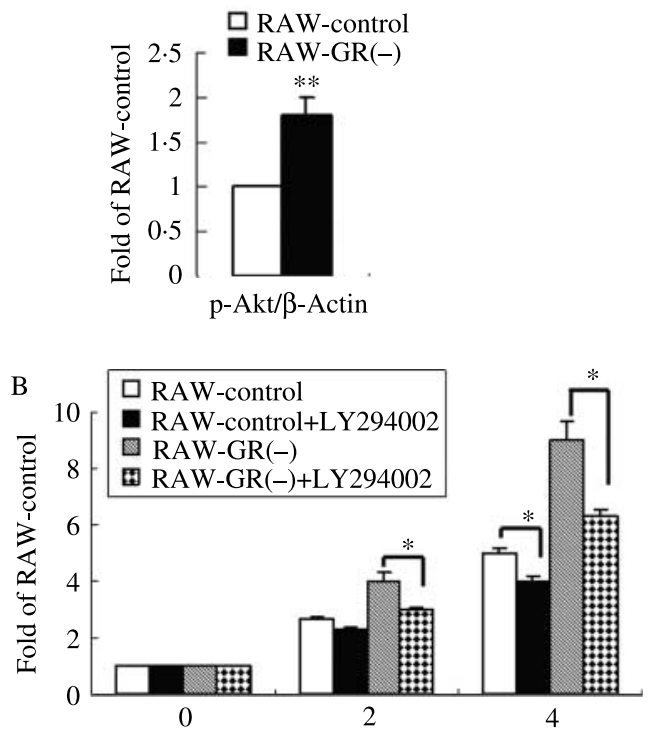

Time (days)

Figure 6 Enhanced expression of phosphorylated Akt is associated with increased proliferation of RAW-GR $(-)$ cells. (A) Phosphorylated Akt (p-Akt) in RAW-GR(-) and RAW-control cells was detected by western blot analysis. These blots are representative of three independent experiments. Using Tanon software, these blots of p-Akt in RAW-GR $(-)$ and RAW-control cells were quantified and normalized against $\beta$-actin. Then the normalized numbers from three independent experiments were compared. The values are expressed as fold of the level of RAW-control, as shown in the bar

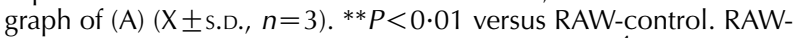
$\mathrm{GR}(-)$ and $\mathrm{RAW}$-control cells were seeded at $3 \times 10^{4}$ cells per well onto a 24-well plate in duplicate and cultured in the medium with or without $25 \mu \mathrm{M}$ LY294002, a specific inhibitor of PI3K/Akt pathway, for 4 days. (B) Proliferation of the cells was monitored by MTT assay $(X \pm$ s.D., $n=3)$. ${ }^{*} P<0 \cdot 05$ versus control cells without LY294002 treatment.

RAW264.7 cells at the same time. A significant decrease in p-Akt protein expression was observed, following incubation of RAW264.7 cells with $10^{-7} \mathrm{M}$ Dex for $4 \mathrm{~h}$ accompanied by an increase in Rhob and RU486, which could partially attenuate the down-regulation of p-Akt by Dex (Fig. 7C), indicating that activation of GR is required for $\mathrm{p}$-Akt reduction. These results demonstrated that Dex inhibited the activity of Akt in RAW264.7 cells and the decrease in Akt activity might be resulted in the increase in Rhob expression of the cells.

\section{Discussion}

In our previous work, we found that the inhibition of GR expression in murine macrophage RAW-GR $(-)$ cells by RNA interference resulted in significant enhancement of cell
A Real-time PCR

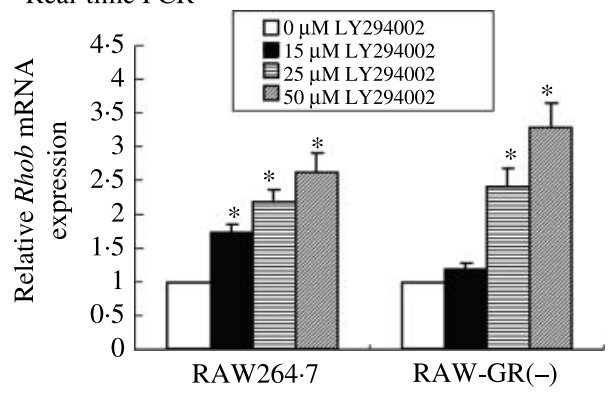

B Western blot

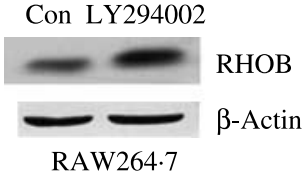

C Western blot

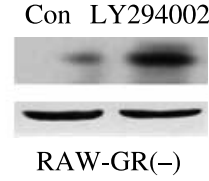

RAW-GR(-)

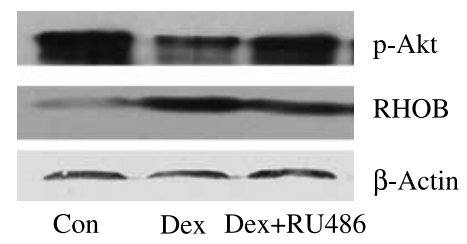

Figure 7 Inhibition of Akt activity by LY294002 or Dex up-regulates Rhob expression. RAW264.7 and RAW-GR $(-)$ cells were treated with LY294002 as indicated, and the expression of Rhob in (A) mRNA and (B) protein levels were detected by real-time PCR and western blot analysis respectively. Similar results were obtained from three independent experiments. ${ }^{*} P<0 \cdot 01$ versus $0 \mu \mathrm{M}$ LY294002. (C) The protein level of p-Akt and RHOB in RAW264.7 cells treated with $10^{-7} \mathrm{M}$ Dex with or without $10^{-6} \mathrm{M}$ RU486 by western blot analysis. Similar results were obtained from three independent experiments.

proliferation (Zhu et al. 2004). These results indicated that GR plays an important antiproliferative role in these macrophage cells. Although it is well known that GR mediates GC action by regulating the expression of target genes (Dondi et al. 2001, Zhu et al. 2004, Bladh et al. 2005), but a few target genes have been known to mediate the antiproliferative effect of GC until now.

Our other previous work found that Rhob could be induced by GC/GR and it at least partially mediated the antiproliferative effect of GC/GR in ovarian cancer HO-8910 cell lines (Chen et al. 2006). In this study, we found that GC also induced the expression of Rhob at both mRNA and protein levels in RAW264.7 cells and activation of GR is required for Rhob induction. The levels of Rhob at both mRNA and protein markedly decreased in RAW-GR(-) and RAW264.7 cells transiently transfected with GR RNA interference vector. Ectopic expression or constructive activation of Rhob by transfection with $R$ hob-wt or Rhob-V14 obviously inhibited the proliferation of RAW-GR $(-)$ cell. Furthermore, 
inhibition of the expression or activity of Rhob by transfection with $R$ hob-RNAi or Rhob-N19 resulted in a significant increase in cell proliferation of parental RAW264.7 cells, while increase in the expression or activity of Rhob by transfection with Rhob-wt or Rhob-V14 inhibited the proliferation. These results demonstrated that $R$ hob negatively regulated the proliferation of RAW264.7 cells and down-regulation of $R$ hob expression was associated with increased proliferation in RAW-GR $(-)$ cell. It needs to be mentioned that Rhob expression and the proliferation of RAW-GR $(-)$ cells showed no significant difference between culture in RPMI 1640 medium containing 10\% heat-inactivated fetal bovine serum and $10 \%$ charcoal-stripped serum (deprived of GC). The data indicated that the presence or absence of GC had no effect on $R$ hob expression when knock down of GR in RAW-GR (-) cells, while GC promoted the expression of Rhob remarkably in RAW264.7 cells through GR mediation. Therefore, we speculated that $R$ hob expression might be mainly regulated by GR in an agonist-independent way.

How GR up-regulates the expression of Rhob remains unknown. It is well known that GR up-regulates gene transcription usually through direct binding to GRE in the promoter region of target genes, where it serves to recruit other proteins, such as coactivators involved in transcription (Zhu et al. 2004, Bladh et al. 2005). However, our previous work demonstrated that the activity of a luciferase reporter gene containing a $\sim 1.9 \mathrm{~kb}(-1756 /+111)$ fragment of the promoter sequence of human $R h o b$ gene was not induced by GC/GR in ovarian cancer HO-8910 cells, indicating that there was no functional GRE in this promoter region $(-1765 /+$ 111; Chen et al. 2006). We also analyzed a $5 \mathrm{~kb}$ fragment of the promoter sequence upstream of the transcriptional start site of the Rhob gene, and found no consensus GRE (Chen et al. 2006). In recent years, evidence has emerged that besides its role in directly regulating gene expression, GR could modulate the activity of several cytoplasmic protein kinases, such as Akt (Leis et al. 2004, Chrysis et al. 2005, Nuutinen et al. 2006), MAPK3/1, and MAPK14 (Miller et al. 2005, Qin et al. 2005), to play its important roles in regulating cell proliferation, differentiation, and apoptosis. Leis et al. (2004) reported that GR inhibited PI3K and Akt activity, which constituted a major mechanism underlying the antitumor effect of GC in skin. Reports also showed that GC/GR induces apoptosis in proliferative chondrocytes and a human follicular lymphoma cell line through suppression of the Akt-PI3K signaling pathway (Chrysis et al. 2005, Nuutinen et al. 2006). In this study, we also found that except for the down-regulation of Rhob, the activity of Akt was enhanced in RAW-GR $(-)$ cells. Furthermore, Dex decreased the expression of p-Akt accompanied with an increase in Rhob. Therefore, it is interesting to know whether the increased proliferation of RAW-GR $(-)$ cells is related to the down-regulation of Rhob resulted from activation of Akt. Treatment of RAW-GR (-) and RAWcontrol cells with $25 \mu \mathrm{M}$ LY294002, a specific inhibitor of $\mathrm{PI} 3 \mathrm{~K} / \mathrm{Akt}$ pathway, resulted in a more obvious growth repression and a more obvious up-regulation of $R$ hob expression in RAW-GR $(-)$ cells than that in RAW-control cells, though the effect could be seen in both the two cells. These results indicated that inhibition of GR expression in RAW-GR (-) cells leads to activation of Akt, and the latter subsequently downregulates the expression of Rhob, which results in the faster growth of the cells. Jiang et al. $(2004 a, b)$ also reported that activated Akt by EGFR-R as signaling suppresses $R$ hob promoter activity by a PI3K/Akt-dependent mechanism.

It has been reported that Akt regulated cell growth progress by controlling the expression and activity of multiple cell cycle regulatory proteins, such as cyclin D1, cyclin E, CDK2, CDK4, and cyclin-dependent kinase inhibitors CDKN1B and CDKN1A (Shukla \& Gupta 2007, Lee et al. 2008, Seo et al. 2008). Wu et al. (2008) reported that PRKCA, a regulator of cell proliferation, was highly expressed in the poorly differentiated hepatocellular carcinoma cell lines and the reduction in PRKCA expression resulted in a decrease in the growth rate and the level of cyclin D1 in these cells. In our previous study, we demonstrated that increased proliferation of RAW-GR $(-)$ cells was associated with an increase in PRKCA and decrease in CDKN1B (Zhu et al. 2004) and with an increase in CDK2, cyclin D1, and cyclin B1 (unpublished). These results suggest that the role of GR on cell cycle regulatory proteins may be through regulating the expression of Akt and PRKCA in RAW264.7 cells, but it needs further study.

In summary, our experiments demonstrate that Rhob plays an important role in the antiproliferative effect of GR on RAW264.7 cells by GR $\rightarrow$ Akt $\rightarrow$ Rhob pathway and Rhob negatively regulated the proliferation of RAW264.7 cells.

\section{Declaration of interest}

The authors declare that there is no conflict of interest that could be perceived as prejudicing the impartiality of the research reported.

\section{Funding}

This work was supported by grants from the National Natural Science Foundation of China (No. 30300128).

\section{Acknowledgements}

We gratefully thank Dr G.C. Prendergast for providing the constitutively active Rhob-G14V, dominant negative Rhob-T19N, and wild-type Rhob cDNA plasmids.

\section{References}

Adnane J, Muro-Cacho C, Mathews L, Sebti SM \& Munoz-Antonia T 2002 Suppression of rho B expression in invasive carcinoma from head and neck cancer patients. Clinical Cancer Research 8 2225-2232.

Bladh LG, Lidén J, Pazirandeh A, Rafter I, Dahlman-Wright K, Nilsson S \& Okret S 2005 Identification of target genes involved in the antiproliferative effect of glucocorticoids reveals a role for nuclear factor- $(\kappa) B$ repression. Molecular Endocrinology 19 632-643.

Brummer E, Choi JH \& Stevens DA 2005 Interaction between conidia, lung macrophages, immunosuppressants, proinflammatory cytokines and transcriptional regulation. Medical Mycology 43 S177-S179. 
Chen YX, Li ZB, Diao F, Cao DM, Fu CC \& Lu J 2006 Up-regulation of RhoB by glucocorticoids and its effects on the cell proliferation and NF- $\kappa B$ transcriptional activity. Journal of Steroid Biochemistry and Molecular Biology 101 179-187.

Chrysis D, Zaman F, Chagin AS, Takigawa M \& Savendahl L 2005 Dexamethasone induces apoptosis in proliferative chondrocytes through activation of caspases and suppression of the Akt-phosphatidylinositol 3'-kinase signaling pathway. Endocrinology 146 1391-1397.

Distelhorst CW 2002 Recent insights into the mechanism of glucocorticosteroid-induced apoptosis. Cell Death and Differentiation 9 6-19.

Dondi D, Maggi R, Scaccianoce E, Martini L, Motta M \& Poletti A 2001 Expression and role of functional glucocorticoid receptors in the human androgen-independent prostate cancer cell line, DU145. Journal of Molecular Endocrinology 26 185-191.

Forget MA, Desrosiers RR, Del M, Moumdjian R, Shedid D, Berthelet F \& Beliveau R 2002 The expression of rho proteins decreases with human brain tumor progression: potential tumor markers. Clinical and Experimental Metastasis 19 9-15.

Frankfurt O \& Rosen ST 2004 Mechanisms of glucocorticoid-induced apoptosis in hematologic malignancies: updates. Current Opinion in Oncology $16553-563$.

Heitzer MD, Wolf IM, Sanchez ER, Witchel SF \& DeFranco DB 2007 Glucocorticoid receptor physiology. Reviews in Endocrine and Metabolic Disorders 8 321-330.

Huang M \& Prendergast GC 2006 RhoB in cancer suppression. Histology and Histopathology 21 213-218.

Jiang K, Delarue FL \& Sebti SM 2004a EGFR, ErbB2 and Ras but not Src suppress $\mathrm{RhoB}$ expression while ectopic expression of RhoB antagonizes oncogene-mediated transformation. Oncogene 23 1136-1145.

Jiang K, Sun J, Cheng J, Djeu JY, Wei S \& Sebti S 2004b Akt mediates Ras downregulation of $\mathrm{RhoB}$, a suppressor of transformation, invasion, and metastasis. Molecular and Cellular Biology 24 5565-5576.

Kudawara I, Ueda T, Yoshikawa H, Miyama T, Yamamoto T \& Nishizawa Y 2001 In vivo inhibition of tumour growth by dexamethasone in murine osteosarcomas. European Journal of Cancer 37 1703-1708.

Lee JY, Jang KS, Shin DH, Oh MY, Kim HJ, Kim Y \& Kong G 2008 Mel-18 negatively regulates INK4a/ARF-independent cell cycle progression via Akt inactivation in breast cancer. Cancer Research 68 4201-4209.

Leis H, Page A, Ramirez A, Bravo A, Segrelles C, Paramio J, Barettino D, Jorcano JL \& Perez P 2004 Glucocorticoid receptor counteracts tumorigenic activity of Akt in skin through interference with the phosphatidylinositol 3-kinase signaling pathway. Molecular Endocrinology 18 303-311.

Liu AX, Rane N, Liu JP \& Prendergast GC 2001 RhoB is dispensable for mouse development, but it modifies susceptibility to tumor formation as well as cell adhesion and growth factor signaling in transformed cells. Molecular and Cellular Biology 21 6906-6912.

Lloberas J, Soler C \& Celada A 1998 Dexamethasone enhances macrophage colony stimulating factor- and granulocyte macrophage colony stimulating factor-stimulated proliferation of bone marrow-derived macrophages. International Immunology 10 593-599.

Mazieres J, Antonia T, Daste G, Muro-Cacho C, Berchery D, Tillement V, Pradines A, Sebti S \& Favre G 2004 Loss of RhoB expression in human lung cancer progression. Clinical Cancer Research 10 2742-2750.

Miller AL, Webb MS, Copik AJ, Wang Y, Johnson BH, Kumar R \& Thompson EB 2005 p38 Mitogen-activated protein kinase (MAPK) is a key mediator in glucocorticoid-induced apoptosis of lymphoid cells: correlation between p38 MAPK activation and site-specific phosphorylation of the human glucocorticoid receptor at serine 211. Molecular Endocrinology 19 1569-1583.

Nuutinen U, Postila V, Matto M, Eeva J, Ropponen A, Eray M, Riikonen P \& Pelkonen J 2006 Inhibition of PI3-kinase-Akt pathway enhances dexamethasone-induced apoptosis in a human follicular lymphoma cell line. Experimental Cell Research 312 322-330.

Pagniello KB, Bols NC \& Lee LE 2002 Effect of corticosteroids on viability and proliferation of the rainbow trout monocyte/macrophage cell line, RTS11. Fish and Shellfish Immunology 13 199-214.

Prendergast GC 2001 Actin' up: RhoB in cancer and apoptosis. Nature Reviews. Cancer 1 162-168.

Qin JM, Yan HX, Wan XW, Liu SQ, Zeng JZ, Cao HF, Wu MC \& Wang HY 2005 Effects of signal regulatory proteinalpha1 on proliferation of hepatocellular carcinoma: a preliminary study. Hepatobiliary and Pancreatic Diseases International 4 244-248.

Sakai M, Biwa T, Matsumura T, Takemura T, Matsuda H, Anami Y, Sasahara T, Kobori S \& Shichiri M 1999 Glucocorticoid inhibits oxidized LDLinduced macrophage growth by suppressing the expression of granulocyte/macrophage colony-stimulating factor. Atteriosclerosis, Thrombosis, and Vascular Biology 19 1726-1733.

Seo JM, Kim TJ, Jin YR, Han HJ, Ryu CK, Sheen YY, Kim DW \& Yun YP 2008 YSK2821, a newly synthesized indoledione derivative, inhibits cell proliferation and cell cycle progression via the cell cycle-related proteins by regulating phosphatidylinositol-3 kinase cascade in vascular smooth muscle cells. European Journal of Pharmacology 586 74-81.

Shukla S \& Gupta S 2007 Apigenin-induced cell cycle arrest is mediated by modulation of MAPK, PI3K-Akt, and loss of cyclin D1 associated retinoblastoma dephosphorylation in human prostate cancer cells. Cell Cycle 6 1102-1114.

Stamatakis K, Cernuda-Morollon E, Hernandez-Perera O \& Perez-Sala D 2002 Isoprenylation of $\mathrm{RhoB}$ is necessary for its degradation. A novel determinant in the complex regulation of $\mathrm{RhoB}$ expression by the mevalonate pathway. Journal of Biological Chemistry 277 49389-49396.

Wu TT, Hsieh YH, Hsieh YS \& Liu JY 2008 Reduction of PKC alpha decreases cell proliferation, migration, and invasion of human malignant hepatocellular carcinoma. Journal of Cellular Biochemistry 103 9-20.

Yamamoto T, Nishiguchi M, Inoue N, Goto HG, Kudawara I, Ueda T, Yoshikawa H, Tanigaki Y \& Nishizawa Y 2002 Inhibition of murine osteosarcoma cell proliferation by glucocorticoid. Anticancer Research 22 4151-4156.

Zhang J, Zhu J, Bu X, Cushion M, Kinane TB, Avraham H \& Koziel H 2005 $\mathrm{Cdc} 42$ and $\mathrm{RhoB}$ activation are required for mannose receptor-mediated phagocytosis by human alveolar macrophages. Molecular Biology of the Cell 16 824-834.

Zhu XY, Liu YJ, Lu J \& Xu RB 2004 Knockdown of glucocorticoid receptor expression by RNA interference promotes cell proliferation in murine macrophage RAW264.7 cells. Journal of Steroid Biochemistry and Molecular Biology 92 375-382.

Received in final form 14 September 2008 Accepted 26 September 2008 Made available online as an Accepted Preprint 7 October 2008 